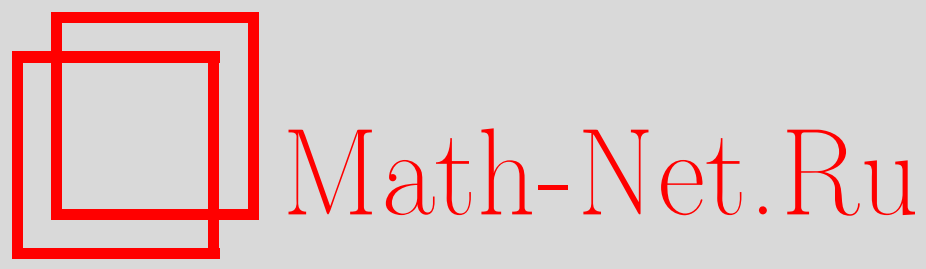

А. Л. Якымив, Предельная теорема для общего числа циклов случайной $A$-подстановки, Теория вероятн. и ее примен., 2007, том 52, выпуск 1, 69-83 DOI: https://doi.org/10.4213/tvp5

Использование Общероссийского математического портала MathNet.Ru подразумевает, что вы прочитали и согласны с пользовательским соглашением

http://www . mathnet.ru/rus/agreement

Параметры загрузки:

IP : 3.82 .47 .9

26 апреля 2023 г., 16:14:40

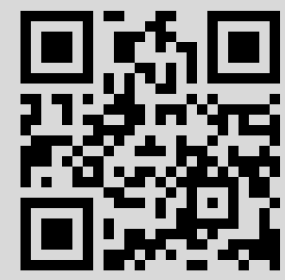




\title{
ПРЕДЕЛЬНАЯ ТЕОРЕМА ДЛЯ ОБЩЕГО ЧИСЛА ЦИКЛОВ СЛУЧАЙНОЙ $A$-ПОДСТАНОВКИ ${ }^{1)}$
}

\begin{abstract}
Пусть $S_{n}$ - симметрическая группа подстановок степени $n$, $A$ - некоторое непустое подмножество множества натуральных чисел $\mathbf{N}$ и $T_{n}=T_{n}(A)-$ совокупность всех подстановок из $S_{n}$, длины циклов которых принадлежат множеству $A$. Подстановки из $T_{n}$ принято называть $A$-подстановками. Пусть $\zeta_{n}$ есть общее число циклов случайной подстановки, равномерно распределенной на $T_{n}$. В статье найден подход, позволяюший исходя из асимптотики числа $A$-подстановок степени $n$ доказывать предельную теорему для $\zeta_{n}$. Предельная теорема для $\zeta_{n}$, полученная здесь, является новой в ряде случаев, когда известна асимптотика числа $A$-подстановок степени $n$, но предельная теорема для $\zeta_{n}$ другими методами еще не доказана. Автором замечено, что в ряде работ различных авторов число $A$-подстановок степени $n$, деленное на $n$ !, представляет собой правильно меняющуюся по Карамата функцию с показателем $\sigma-1$, где $\sigma$ - асимптотическая плотность множества $A$ (положительная). Основной целью настоящей статьи является доказательство предельной теоремы для $\zeta_{n}$ именно при этом предположении, без каких-либо дополнительных ограничений, характерных для предыдуших исследований.
\end{abstract}

Ключевые слова и фразы: асимптотическая плотность множества $A$, логарифмическая плотность множества $A$, случайные $A$ подстановки, общее число циклов $A$-подстановки, правильно меняющиеся функции, медленно меняюшиеся функции, тауберова теорема.

1. Введение. Зафиксируем непустое множество $A \subseteq \mathbf{N}=$ $\{1,2,3, \ldots\}$. A-подстановками называются подстановки, длины циклов которых принадлежат множеству $A$ (см. книгу [28]). Пусть $T_{n}=T_{n}(A)$ есть множество $A$-подстановок степени $n$ и $c_{n}$ - случайная подстановка, равномерно распределенная на $T_{n}$. Через $\zeta_{m n}$ обозначим число циклов подстановки $c_{n}$, имеющих длину $m \in \mathbf{N}$. Ясно, что $\zeta_{m n}=0$ при $m \notin A$.

* Математический институт им. В. А. Стеклова РАН, ул. Губкина, 8, 119991 Москва, ГСП-1, Россия; e-mail: arsen@mi.ras.ru

1) Работа выполнена при поддержке РФФИ (грант № 05-01-00583) и гранта президента РФ по поддержке ведущих научных школ НШ-4129.2006.1. 
Далее, пусть $\zeta_{n}$ - общее число циклов случайной подстановки $c_{n}$, т.е.

$$
\zeta_{n}=\sum_{m \in \mathbf{N}} \zeta_{m n} .
$$

Случайным $A$-подстановкам посвящено большое число работ (см. [1]-[9], [11], [12], [14]-[23], [25], [26], [31]-[38]). Кроме того, имеется пять монографий, содержащих сведения об $A$-подстановках: [10], [13], [27], [28], [39].

Через $|X|$ мы будем везде далее обозначать число элементов конечного множества $X$. В большинстве упомянутых работ для тех или иных классов множеств $A$ установлена асимптотика $\left|T_{n}(A)\right|$ при $n \rightarrow \infty$. Кроме того, в некоторых из них получены предельные теоремы для $\zeta_{m n}$ и $\zeta_{n}$ при фиксированном $m \in A$ и $n \rightarrow \infty$. При этом, как правило, наиболее сложной для доказательства оказывалась предельная теорема для $\zeta_{n}$.

В настоящей статье мы будем предполагать, что

$$
p(n) \equiv \frac{\left|T_{n}(A)\right|}{n !}=n^{\sigma-1} L(n), \quad n \in \mathbf{N},
$$

где функция $L(n)$ медленно меняется на бесконечности [29], а $\sigma \in(0,1]$. Имеется широкий класс примеров множеств $A$, для которых выполнено (1) (см. работы [3], [6], [11], [12], [18]-[20], [23], [32]-[37]), более подробный обзор этих работ содержится в статье [38].

В [20] показано, что из (1) следует существование логарифмической плотности множества $A$, равной $\sigma$, т.е. существование предела

$$
\lim _{n \rightarrow \infty} \frac{1}{\ln n} \sum_{m \in A, m \leqslant n} \frac{1}{m}=\sigma .
$$

Несколько более сильное утверждение получено в недавней работе [38], а именно, в [38] показано, что из (1) следует существование асимптотической плотности множества $A$, равной $\sigma$, т.е. сушествование предела

$$
\lim _{n \rightarrow \infty} \frac{1}{n} \sum_{m \in A, m \leqslant n} 1=\sigma .
$$

(Как известно, из существования асимптотической плотности множества $A$ следует существование логарифмической плотности $A$, но не наоборот - см. [24, раздел 3.1].) Отметим также, что из (3) не следует (1), как это видно на примере множества всех четных натуральных чисел.

В настоящей статье доказана предельная теорема об асимптотической нормальности $\zeta_{n}$ при $n \rightarrow \infty$. Отметим, что ранее предельная теорема для $\zeta_{n}$ доказывалась различными авторами при выполнении условий типа (1), (2), (3) и других. Предлагаемая ниже теорема требует выполнения только условия (1). 
2. Основной результат. Положим при $n \in \mathbf{N}$

$$
l(n)=\sum_{m \in A(n)} \frac{1}{m}
$$

где $A(n)=A \cap[1, n], l(n)=0$ при $A(n)=\varnothing$. Основным результатом настоящей статьи является следующая предельная теорема.

Теорема 1. Пусть выполнено (1). Тогда при $n \rightarrow \infty$ распределение случайной величинь

$$
\zeta_{n}^{\prime}=\frac{\zeta_{n}-l(n)}{\sqrt{\sigma \ln n}}
$$

слабо сходится $\kappa$ стандартному нормальному закону.

Теорема 1 выводится из некоторой модификации тауберовой теоремы 4 статьи [32], а именно, следующей теоремы.

Теорема 2. Пусть для всех $u, v \in(0,1)$ конечна Функиия

$$
A(u, v)=\sum_{m, n \geqslant 0} m^{\alpha-1} a(m, n) u^{m} v^{n}
$$

$(\alpha>1, a(m, n) \geqslant 0)$, причем для произвольньцх $\lambda, \mu>0$

$$
A \frac{e^{-\lambda / t}, e^{-\mu / t}}{r(t)} \longrightarrow \lambda^{-\alpha} \mu^{-\beta} \Gamma(\alpha) \Gamma(\beta)
$$

при $t \rightarrow \infty$, где $\beta>0, r(t)$ - некоторая положительная функиия переменной $t, \Gamma(\cdot)$ - гамма-функиия Эйлера. Если $a(m, n)$ монотонна nо $m$ u nрu $n \rightarrow \infty, m \asymp n, w \geqslant n, w-n=o(n)$

$$
\frac{a(m, w)-a(m, n)}{a(m, w)+a(m, n)} \longrightarrow 0,
$$

то для произвольньи $y, z>0$ nрu $t \rightarrow \infty$

$$
a(t z, t y) \sim r(t) t^{-1-\alpha} y^{\beta-1}
$$

(при нецельх $u, v$ полагаем $a(u, v)=a([u],[v]))$.

3 а м е ч а н и е 1. Для выполнения (7) необходимо и достаточно, чтобы при $n \rightarrow \infty, m \asymp n, w \geqslant n, w-n=o(n)$

$$
\frac{a(m, w)}{a(m, n)} \rightarrow 1 \text {. }
$$

Тем не менее, при доказательстве теоремы 1 нам удобно воспользоваться тауберовым условием именно в форме (7).

В самом деле, из (9) следует, что при $n \rightarrow \infty, m \asymp n, w \geqslant n$, $w-n=o(n)$

$$
\frac{a(m, w)-a(m, n)}{a(m, n)} \longrightarrow 0,
$$


откуда следует (7) ввиду неотрицательности $a(m, w)$. Обратно, пусть имеет место (7). Тогда при $n \rightarrow \infty, m \asymp n, w \geqslant n, w-n=o(n)$

$$
\frac{a(m, w)-a(m, n)}{a(m, w)+a(m, n)}=\frac{a(m, w) / a(m, n)-1}{a(m, w) / a(m, n)+1} \longrightarrow 0 .
$$

Из последнего соотношения следует (9), так как функция $g(z)$, обратная к функции $f(z)=(z-1) /(z+1)$, непрерывна в нуле и $g(0)=1$.

Зафиксируем произвольное неотрицательное $x$ и положим при $m, n \in \mathbf{N} \cup\{0\}$

$$
a(m, n)=p(n) \mathbf{E} \exp \left(\frac{\zeta_{n} x}{\sqrt{l(m)}}\right)
$$

где $p(0)=1, l(0)=0$ (при $l(m)=0$ будем считать $a(m, n)=0)$. Ключом для использования тауберовой теоремы 2 при доказательстве теоремы 1 является следующая лемма.

Лемма 1. Предположим, что выполнено соотношение (1). Тогда для чисел $a(m, n)$, определенньх равенством (10), справедливо соотношение (7).

3. Доказательства. Д о ка за т е л с т в о т е о е мы 2. В работе [32] показано, что из (6) и монотонности $a(m, n)$ по $m$ следует, что для произвольных $y, z>0$ при $m=[t z]$ и $t \rightarrow \infty$

$$
\sum_{w=0}^{[t y]} a(m, w) \frac{t^{\alpha}}{r(t)} \longrightarrow \frac{y^{\beta}}{\beta}
$$

откуда для произвольного $\delta>0$ при $t \rightarrow \infty$

$$
\sum_{w=[t y]+1}^{[t y(1+\delta)]} a(m, w) \frac{t^{\alpha}}{r(t)} \longrightarrow \frac{y^{\beta}}{\beta}\left((1+\delta)^{\beta}-1\right) .
$$

Зафиксируем произвольное $\varepsilon \in(0,1)$. Пусть положительные константы $\delta$ и $t_{0}$ таковы, что при $t \geqslant t_{0}$ и $0 \leqslant w-[t y] \leqslant \delta t y+1$

$$
-\varepsilon(a(m, w)+a(m, n)) \leqslant a(m, w)-a(m, n) \leqslant \varepsilon(a(m, w)+a(m, n)),
$$

где $n=[t y]$, откуда

$$
a(m, n) \geqslant a(m, w) \frac{1-\varepsilon}{1+\varepsilon} .
$$

Отсюда при $M=[t y(1+\delta)]-[t y]$

$$
a(m, n) M \geqslant \frac{1-\varepsilon}{1+\varepsilon} \sum_{w=[t y]+1}^{[t y(1+\delta)]} a(m, w)
$$

или же

$$
a(m, n) \geqslant \frac{1-\varepsilon}{M(1+\varepsilon)} \sum_{w=[t y]+1}^{[t y(1+\delta)]} a(m, w) .
$$


Из последнего неравенства с использованием (12) выводим, что

$$
\begin{aligned}
\liminf _{t \rightarrow \infty} \frac{a(m, n) t^{1+\alpha}}{r(t)} & \geqslant \frac{1-\varepsilon}{1+\varepsilon} \lim _{t \rightarrow \infty} \frac{t}{[t y(1+\delta)]-[t y]} \sum_{w=[t y]+1}^{[t y(1+\delta)]} a(m, w) \frac{t^{\alpha}}{r(t)} \\
& =\frac{1-\varepsilon}{1+\varepsilon} \frac{1}{\delta y} \frac{y^{\beta}}{\beta}\left((1+\delta)^{\beta}-1\right) .
\end{aligned}
$$

Поскольку левая часть выписанного неравенства не зависит от $\varepsilon$ и $\delta$, то, устремляя в правой части $\varepsilon$ и $\delta$ к нулю, убеждаемся в том, что

$$
\liminf _{t \rightarrow \infty} \frac{a(m, n) t^{1+\alpha}}{r(t)} \geqslant y^{\beta-1} .
$$

Из неравенств (13) следует также, что при $t \geqslant t_{0}$ и $0 \leqslant w-[t y] \leqslant \delta t y+1$

$$
a(m, n) \leqslant a(m, w) \frac{1+\varepsilon}{1-\varepsilon},
$$

откуда

$$
a(m, n) \leqslant a(m, w) \frac{1+\varepsilon}{M(1-\varepsilon)} \sum_{w=[t y]+1}^{[t y(1+\delta)]} a(m, w) .
$$

Поэтому

$$
\limsup _{t \rightarrow \infty} \frac{a(m, n) t^{1+\alpha}}{r(t)} \leqslant \frac{1+\varepsilon}{1-\varepsilon} \frac{1}{\delta y} \frac{y^{\beta}}{\beta}\left((1+\delta)^{\beta}-1\right),
$$

или, в силу произвольности $\varepsilon$ и $\delta$,

$$
\limsup _{t \rightarrow \infty} \frac{a(m, n) t^{1+\alpha}}{r(t)} \leqslant y^{\beta-1} .
$$

Из (14) и (15) следует (8). Теорема 2 доказана.

Д о к а з а т ел ь с т в о л е м м ы 1 . Пусть $m \geqslant \min A$. Положим $h(m)=\exp (x / \sqrt{l(m)})$. Согласно $[28,(0.14)]$, для произвольного $s \in[0,1)$

$$
\sum_{n \geqslant 0} a(m, n) s^{n}=\exp \left(f(s)+\sum_{i \in A}(h(m)-1) \frac{s^{i}}{i}\right),
$$

где

$$
f(s)=\sum_{i \in A} \frac{s^{i}}{i}
$$

В силу (16) $a(m, n)=\operatorname{coef}_{s^{n}} \exp \left(f(s)+\sum_{i \in A}(h(m)-1) s^{i} / i\right)$. Согласно $[3,(6.3)], p(l)=\operatorname{coef}_{s^{l}} \exp (f(s))$ при $l \in \mathbf{N} \cup\{0\}$ (это следует также из формулы $(16)$ при $x=0)$. Поэтому

$$
a(m, n)=\operatorname{coef}_{s^{n}} \sum_{l=0}^{\infty} p(l) s^{l} \sum_{k=0}^{\infty} \frac{(h(m)-1)^{k}}{k !}\left(\sum_{i \in A} \frac{s^{i}}{i}\right)^{k}
$$




$$
\begin{aligned}
& =\operatorname{coef}_{s^{n}} \sum_{k=0}^{\infty} \frac{(h(m)-1)^{k}}{k !} \sum_{l=0}^{\infty} p(l) s^{l}\left(\sum_{i \in A} \frac{s^{i}}{i}\right)^{k} \\
& =p(n)+\sum_{k=1}^{\infty} \frac{(h(m)-1)^{k}}{k !} S_{k}(n)
\end{aligned}
$$

где

$$
S_{k}(n)=\sum_{i_{1}, \ldots, i_{k} \in A} \frac{1}{i_{1} \cdots i_{k}} p\left(n-i_{1}-\cdots-i_{k}\right)
$$

(мы полагаем $p(r)=0$ при $r<0$ ). Поскольку $S_{k}(n)=0$ при $k>n$, то мы имеем:

$$
a(m, n)=p(n)+\sum_{k=1}^{n} \frac{(h(m)-1)^{k}}{k !} S_{k}(n)=p(n)+\sum_{k=1}^{n} b_{k}(m, n)
$$

где

$$
b_{k}(m, n)=\frac{(h(m)-1)^{k}}{k !} S_{k}(n)
$$

Из (2) и (4) следует, что

$$
l(n) \sim \sigma \ln n \quad(n \rightarrow \infty) .
$$

Для данной последовательности $r(n)=w(n)-n$ подберем такую последовательность $g(n)>0$, чтобы было выполнено

$$
g(n)=o(n), \quad \frac{r(n)}{g(n)} \rightarrow 0 \quad(n \rightarrow \infty) .
$$

Так как $r(n)=o(n)$ при $n \rightarrow \infty$, то такой выбор возможен. Оценим сверху

$$
I_{1}(k, n)=\sum_{i_{1}, \ldots, i_{k} \in A, \sum_{j=1}^{k} i_{j} \in[n-g(n), n]} \frac{1}{i_{1} \cdots i_{k}} p\left(n-i_{1}-\cdots-i_{k}\right) .
$$

Поскольку $\sum_{j=1}^{k} i_{j} \geqslant v(n) \equiv n-g(n)$, то для некоторого $j=1, \ldots, n$ справедливо неравенство $i_{j} \geqslant v(n) / k$. Поэтому

$$
I_{1}(k, n) \leqslant k \sum_{i_{1}, \ldots, i_{k} \in A, \sum_{j=1}^{k}} \frac{1}{i_{j} \in[n v(n), n], i_{k} \geqslant v(n) / k} p\left(n-i_{1}-\cdots-i_{k}\right) .
$$

Введем замену переменных

$$
y=n-i_{1}-\cdots-i_{k} \quad\left(i_{k}=n-i_{1}-\cdots-i_{k-1}-y\right) .
$$


Так как $i_{k} \geqslant v(n) / k$, то, согласно $(24)$,

$$
\begin{aligned}
I_{1}(k, n) & \leqslant \frac{k^{2}}{v(n)} \sum_{y=0}^{g(n)} p(y) \sum_{i_{1}, \ldots, i_{k-1} \in A, \sum_{j=1}^{k-1} i_{j} \leqslant n-1} \frac{1}{i_{1} \cdots i_{k-1}} \\
& \leqslant \frac{k^{2}}{v(n)} \sum_{y=0}^{g(n)} p(y) \sum_{i_{1}, \ldots, i_{k-1} \in A(n)} \frac{1}{i_{1} \cdots i_{k-1}} \\
& =\frac{k^{2}}{v(n)} \sum_{y=0}^{g(n)} p(y)(l(n))^{k-1}=\frac{k^{2}}{v(n)} O(g(n) p(g(n)))(l(n))^{k-1}
\end{aligned}
$$

при $n \rightarrow \infty$. Поскольку $n p(n)$ правильно меняется на бесконечности с положительным показателем $\sigma$ и $g(n)=o(n)(n \rightarrow \infty)($ см. (1) и (22)), то

$$
g(n) p(g(n))=o(n p(n)) \quad(n \rightarrow \infty) .
$$

Из (22), (25) и (26) следует, что при $k \leqslant n$

$$
I_{1}(k, n) \leqslant \delta(n) \frac{k^{2}}{v(n)} n p(n)(l(n))^{k-1}=\delta_{1}(n) k^{2} p(n)(l(n))^{k-1},
$$

где $\delta(n) \rightarrow 0$ и $\delta_{1}(n) \rightarrow 0$ при $n \rightarrow \infty$. Зафиксируем $c \in(0,1)$ и положим

$$
I_{2}(k, n)=\sum_{i_{1}, \ldots, i_{k} \in A, \sum_{j=1}^{k} i_{j} \in(n c, n]} \frac{1}{i_{1} \cdots i_{k}} p\left(n-i_{1}-\cdots-i_{k}\right) .
$$

Проводя точно такие же оценки, как и с суммой $I_{1}(k, n)$, убеждаемся в том, что при $n \rightarrow \infty$ и $k \leqslant n$

$$
I_{2}(k, n)=O\left(k^{2} p(n)(l(n))^{k-1}\right),
$$

причем константа в «O» не зависит от $k$. Положим

$$
I_{3}(k, n)=\sum_{i_{1}, \ldots, i_{k} \in A, \sum_{j=1}^{k} i_{j} \leqslant c n} \frac{1}{i_{1} \cdots i_{k}} p\left(n-i_{1}-\cdots-i_{k}\right) .
$$

Поскольку в наших условиях суммирования $n>n-i_{1}-\cdots-i_{k} \geqslant n(1-c)$, то в силу (1) при $n \rightarrow \infty$ и $k \leqslant n$

$$
I_{3}(k, n)=O(p(n)) \sum_{i_{1}, \ldots, i_{k} \in A, \sum_{j=1}^{k} i_{j} \leqslant c n} \frac{1}{i_{1} \cdots i_{k}},
$$

причем константа в «O» не зависит от $k$. Как и ранее,

$$
\sum_{i_{1}, \ldots, i_{k} \in A ; \sum_{j=1}^{k} i_{j} \leqslant c n} \frac{1}{i_{1} \cdots i_{k}} \leqslant \sum_{i_{1}, \ldots, i_{k} \in A(n)} \frac{1}{i_{1} \cdots i_{k}}=(l(n))^{k} .
$$

Из (31) и (32) следует, что при $n \rightarrow \infty$ и $k \leqslant n$

$$
I_{3}(k, n)=O\left(p(n)(l(n))^{k}\right),
$$


причем константа в «O» не зависит от $k$. Далее, оценим снизу при $k \leqslant c n$ и достаточно больших $n$

$$
\begin{aligned}
S_{k}(n) & =\sum_{i_{1}, \ldots, i_{k} \in A} \frac{1}{i_{1} \cdots i_{k}} p\left(n-i_{1}-\cdots-i_{k}\right) \\
& \geqslant \sum_{i_{1}, \ldots, i_{k} \in A, \sum_{j=1}^{k} i_{j} \leqslant c n} \frac{1}{i_{1} \cdots i_{k}} p\left(n-i_{1}-\cdots-i_{k}\right) .
\end{aligned}
$$

Так как в последней сумме $n>n-i_{1}-\cdots-i_{k} \geqslant n(1-c)$, то в силу (1) для достаточно больших $n \geqslant n_{1}>0$ и некоторой постоянной $c_{1}>0$, не зависящей от $k$,

$$
\begin{aligned}
S_{k}(n) & \geqslant c_{1} p(n) \sum_{i_{1}, \ldots, i_{k} \in A, \sum_{j=1}^{k} i_{j} \leqslant c n} \frac{1}{i_{1} \cdots i_{k}} \\
& \geqslant c_{1} p(n) \sum_{i_{1}, \ldots, i_{k} \in A(c n / k)} \frac{1}{i_{1} \cdots i_{k}}=c_{1} p(n)\left(l\left(\frac{c n}{k}\right)\right)^{k} .
\end{aligned}
$$

(Для функций $F(t)$, определенных при $t \in \mathbf{N}$, при нецелых $t>1$ мы полагаем $F(t)=F([t])$.) Заметим, что

$$
\begin{aligned}
l(n)-l\left(\frac{c n}{k}\right) & =\sum_{i=[c n / k]+1, i \in A}^{n} \frac{1}{i} \leqslant \sum_{i=[c n / k]+1}^{n} \frac{1}{i} \\
& \leqslant \ln n-\ln \left(\left[\frac{c n}{k}\right]\right) \leqslant \ln \left(\frac{n}{c n / k-1}\right) \\
& =\ln \left(\frac{1}{c / k-1 / n}\right)=\ln \left(\frac{k}{c-k / n}\right) \\
& \leqslant \ln \left(\frac{k}{c / 2}\right)=\ln k+c_{2}
\end{aligned}
$$

при $k \leqslant c n / 2$, где $c_{2}=\ln (2 / c)$. Из (35) следует, что при $k \leqslant c n / 2$

$$
\left(l\left(\frac{c n}{k}\right)\right)^{k} \geqslant(l(n))^{k}\left(1-\frac{\ln k+c_{2}}{l(n)}\right)^{k} .
$$

В силу (21) существует $n_{2}>n_{1}$ такое, что при $n \geqslant n_{2}$ выполнено неравенство $l(n) \geqslant \sigma_{1} \ln n$, где постоянная $\sigma_{1} \in(0, \sigma)$. Поэтому из $(36)$ следует, что при $n \geqslant n_{2}$ и $k \leqslant c n / 2$

$$
\left(l\left(\frac{c n}{k}\right)\right)^{k} \geqslant(l(n))^{k}\left(1-\frac{\ln k+c_{2}}{\sigma_{1} \ln n}\right)^{k}=(l(n))^{k} \exp \left(k \ln \left(1-\frac{\ln k+c_{2}}{\sigma_{1} \ln n}\right)\right) .
$$

Заметим, что для произвольной постоянной $M>0$ при $k \leqslant M \sqrt{\ln n}$

$$
k \ln \left(1-\frac{\ln k+c_{2}}{\sigma_{1} \ln n}\right)=O\left(\frac{k \ln k+k c_{2}}{\ln n}\right)=o(1) \quad(n \rightarrow \infty) .
$$


Стало быть, существует $n_{3}>n_{2}$ такое, что при $n \geqslant n_{3}$ и $k \leqslant M \sqrt{\ln n}$

$$
\left(l\left(\frac{c n}{k}\right)\right)^{k} \geqslant c_{3}(l(n))^{k}
$$

где постоянная $c_{3} \in(0,1)$ не зависит от $k$. Из (34) и (37) следует, что при $n \geqslant n_{3}$ и $k \leqslant M \sqrt{\ln n}$

$$
S_{k}(n) \geqslant c_{4} p(n)(l(n))^{k}
$$

где $c_{4}=c_{1} c_{3}$. Далее, покажем, что для некоторой постоянной $M>0$ при $n \rightarrow \infty$ и $m \asymp n$

$$
\sum_{k>M \sqrt{\ln n}} b_{k}(m, n)=o(p(n)) .
$$

В самом деле, из (29) и (33) следует, что при $n \rightarrow \infty$ и $k \leqslant n$

$$
S_{k}(n)=I_{2}(k, n)+I_{3}(k, n)=O\left(k^{2} p(n)(l(n))^{k}\right),
$$

где постоянная в «O» не зависит от $k$. Значит, существуют такие $n_{4}>$ $n_{3}$ и постоянная $c_{5} \in(0, \infty)$, что при $n \geqslant n_{4}$ и $k \leqslant n$

$$
S_{k}(n) \leqslant c_{5} k^{2} p(n)(1+\ln n)^{k}
$$

в силу неравенства $l(n)=\sum_{i \in A(n)} 1 / i \leqslant \sum_{i=1}^{n} 1 / i<1+\ln n$. Далее, при $n \rightarrow \infty$ и $m \asymp n$, согласно $(21)$,

$$
\begin{aligned}
h(m)-1 & =\exp \left(\frac{x}{\sqrt{l(m)}}\right)-1=(1+o(1)) \frac{x}{\sqrt{l(m)}} \\
& =(1+o(1)) \frac{x}{\sqrt{\sigma \ln m}}=(1+o(1)) \frac{x}{\sqrt{\sigma} \sqrt{\ln n}} .
\end{aligned}
$$

Следовательно, для произвольных постоянных $s_{1}$ и $s_{2}, 0<s_{1}<s_{2}<\infty$, существует такое $n_{5}>n_{4}$, что при $n \geqslant n_{5}$ и $m \in\left[s_{1} n, s_{2} n\right]$

$$
h(m)-1 \leqslant \frac{x+1}{\sqrt{\sigma} \sqrt{\ln n}} .
$$

В силу (41) и (42), при $n \geqslant n_{5}$ и $m \in\left[s_{1} n, s_{2} n\right]$

$$
\begin{aligned}
b_{k}(m, n) & =\frac{(h(m)-1)^{k}}{k !} S_{k}(n) \leqslant c_{5} k^{2}\left(\frac{x+1}{\sqrt{\sigma}}\right)^{k} \frac{1}{k !(\ln n)^{k / 2}}(2 \ln n)^{k} p(n) \\
& =c_{5} k^{2} c_{6}^{k} \frac{(\sqrt{\ln n})^{k}}{k !} p(n) \equiv H(k, n),
\end{aligned}
$$

где $c_{6}=2(x+1) / \sqrt{\sigma}$. Учитывая формулу Стирлинга для $k !$, получаем, что

$$
\begin{aligned}
H(k, n) & =(1+\eta(k)) c_{7} k^{3 / 2}\left(e c_{6}\right)^{k}\left(\frac{\sqrt{\ln n}}{k}\right)^{k} p(n) \\
& =(1+\eta(k)) c_{7} k^{3 / 2}\left(\frac{e c_{6} \sqrt{\ln n}}{k}\right)^{k} p(n),
\end{aligned}
$$


где $c_{7}=c_{5} / \sqrt{2 \pi}$, а $\eta(k) \rightarrow 0$ при $k \rightarrow \infty$. Из (43) и (44) выводим, что при $m \in\left[s_{1} n, s_{2} n\right]$ и $n \rightarrow \infty$

$$
\sum_{k>e^{2} c_{6} \sqrt{\ln n}} b_{k}(m, n)=O(p(n)) \sum_{k>e^{2} c_{6} \sqrt{\ln n}} k^{3 / 2} e^{-k}=o(p(n)),
$$

откуда следует (39) с $M=e^{2} c_{6}$. В силу (19) и (39) для доказательства (7) достаточно показать, что при $n \rightarrow \infty, m \asymp n, w \geqslant n, w-n=o(n)$

$$
\frac{v(m, w)-v(m, n)}{v(m, w)+v(m, n)} \longrightarrow 0,
$$

где

$$
v(m, n)=p(n)+\sum_{k=1}^{[M \sqrt{\ln n]}} b_{k}(m, n) .
$$

Докажем (45). Положим

$$
I_{4}(k, n)=\sum_{i_{1}, \ldots, i_{k} \in A, \sum_{j=1}^{k} i_{j}<n-g(n)} \frac{1}{i_{1} \cdots i_{k}} p\left(n-i_{1}-\cdots-i_{k}\right) .
$$

Полагая $a=\sum_{j=1}^{k} i_{j}$, мы имеем, согласно (1), при $r=r(n)=w(n)-n$

$$
\begin{aligned}
& \frac{p(n-a+r)-p(n-a)}{p(n-a)}=\frac{p(n-a+r)}{p(n-a)}-1 \\
& \quad=\left(1+\frac{r}{n-a}\right)^{\sigma-1} \frac{L(n-a+r)}{L(n-a)}-1 \\
& \quad=\left(1+\frac{r}{n-a}\right)^{\sigma-1}(1+o(1))=o(1)+(1+o(1)) \frac{r(\sigma-1)}{n-a}=o(1)
\end{aligned}
$$

равномерно по $n-a>g(n)$, так как по условию (11) $r(n)=o(g(n))$ при $n \rightarrow \infty$. Точно так же мы убеждаемся, что

$$
\frac{p(n+r)-p(n)}{p(n)}=o(1)
$$

при $n \rightarrow \infty$ и $r=o(n)$. Из (47) и (48) следует, что

$$
\left|I_{4}(k, w)-I_{4}(k, n)\right| \leqslant \varepsilon(r, n) I_{4}(k, n) \leqslant \varepsilon(r, n) S_{k}(n),
$$

где $\varepsilon(r, n) \rightarrow 0$ при $n \rightarrow \infty$ и $r=o(n)$. Далее,

$$
I_{1}(k, w)-I_{1}(k, n) \geqslant-I_{1}(k, n) .
$$

Согласно $(21)$ и $(27)$, при $k \leqslant M \sqrt{\ln n}$

$$
I_{1}(k, n) \leqslant M^{2} \delta_{1}(n) p(n)(l(n))^{k-1} \ln n=\delta_{2}(n) p(n)(l(n))^{k},
$$

где $\delta_{2}(n) \rightarrow 0$ при $n \rightarrow \infty$. Из (38) и (52) следует, что при $n \geqslant n_{3}$ и $k \leqslant M \sqrt{\ln n}$

$$
I_{1}(k, n) \leqslant \delta_{3}(n) S_{k}(n)
$$


где $\delta_{3}(n) \rightarrow 0$ при $n \rightarrow \infty$. Из (50), (51) и (53) получаем, что при $n \geqslant n_{3}$ и $k \leqslant M \sqrt{\ln n}$

$$
\begin{aligned}
S_{k}(w)-S_{k}(n) & =\left(I_{4}(k, w)-I_{4}(k, n)\right)+\left(I_{1}(k, w)-I_{1}(k, n)\right) \\
& \geqslant-\left(\varepsilon(r, n)+\delta_{3}(n)\right) S_{k}(n)=-\varepsilon_{1}(r, n) S_{k}(n)
\end{aligned}
$$

где

$$
\varepsilon_{1}(r, n) \geqslant 0, \quad \varepsilon_{1}(r, n) \rightarrow 0
$$

при $n \rightarrow \infty$ и $r / n \rightarrow 0$. Из $(20)$, (46) и (54) следует, что при $n \geqslant n_{3}$

$$
\begin{aligned}
v(m, w)-v(m, n) & =p(w)-p(n)+\sum_{k=1}^{[M \sqrt{\ln n}]} \frac{(h(m)-1)^{k}}{k !}\left(S_{k}(w)-S_{k}(n)\right) \\
& \geqslant p(w)-p(n)-\varepsilon_{1}(r, n) \sum_{k=1}^{[M \sqrt{\ln n}]} \frac{(h(m)-1)^{k}}{k !} S_{k}(n) \\
& \geqslant-\varepsilon_{2}(r, n)\left(p(n)+\sum_{k=1}^{[M \sqrt{\ln n]}} b_{k}(m, n)\right) \\
& =-\varepsilon_{2}(r, n) v(m, n) \geqslant-\varepsilon_{2}(r, n)(v(m, w)+v(m, n)),
\end{aligned}
$$

где

$$
\varepsilon_{2}(r, n) \geqslant 0, \quad \varepsilon_{2}(r, n) \rightarrow 0
$$

при $n \rightarrow \infty$ и $r / n \rightarrow 0$ в силу (49) и (55). Из (56) и (57) следует, что при $n \rightarrow \infty, m \asymp n, w \geqslant n, w-n=o(n)$

$$
\liminf \left(\frac{v(m, w)-v(m, n)}{v(m, w)+v(m, n)}\right) \geqslant 0 .
$$

Для получения аналогичного неравенства сверху вместо (51) используем соотношение

$$
I_{1}(k, w)-I_{1}(k, n) \leqslant I_{1}(k, w) .
$$

Из (53) следует, что при $w \geqslant n_{3}$ и $k \leqslant M \sqrt{\ln n}$

$$
I_{1}(k, w) \leqslant \delta_{3}(w) S_{k}(w)
$$

где $\delta_{3}(w) \rightarrow 0$ при $w \rightarrow \infty$. Из (50), (59) и (60) получаем, что при $w \geqslant n \geqslant n_{3}$ и $k \leqslant M \sqrt{\ln n}$

$$
\begin{aligned}
S_{k}(w)-S_{k}(n) & =\left(I_{4}(k, w)-I_{4}(k, n)\right)+\left(I_{1}(k, w)-I_{1}(k, n)\right) \\
& \leqslant \varepsilon(r, n) S_{k}(n)+\delta_{3}(w) S_{k}(w) .
\end{aligned}
$$

Из (20), (46) и (61) следует, что при $w \geqslant n \geqslant n_{3}$

$$
v(m, w)-v(m, n)=p(w)-p(n)+\sum_{k=1}^{[M \sqrt{\ln n}]} \frac{(h(m)-1)^{k}}{k !}\left(S_{k}(w)-S_{k}(n)\right)
$$




$$
\begin{aligned}
& \leqslant p(w)-p(n)+\varepsilon_{1}(r, n) \sum_{k=1}^{[M \sqrt{\ln n}]} \frac{(h(m)-1)^{k}}{k !} S_{k}(n) \\
& \quad+\delta_{3}(w) \sum_{k=1}^{[M \sqrt{\ln n]}} \frac{(h(m)-1)^{k}}{k !} S_{k}(w) .
\end{aligned}
$$

В силу (46) и (49) мы имеем:

$$
\begin{aligned}
& p(w)-p(n)+\varepsilon_{1}(r, n) \sum_{k=1}^{[M \sqrt{\ln n}]} \frac{(h(m)-1)^{k}}{k !} S_{k}(n) \\
& \quad \leqslant \varepsilon_{3}(r, n)\left(p(n)+\sum_{k=1}^{[M \sqrt{\ln n]}} \frac{(h(m)-1)^{k}}{k !} S_{k}(n)\right)=\varepsilon_{3}(r, n) v(m, n),
\end{aligned}
$$

где

$$
\varepsilon_{3}(r, n) \rightarrow 0
$$

при $n \rightarrow \infty$ и $r / n \rightarrow 0$. Далее, так как $w \geqslant n$, то

$$
\begin{aligned}
& \sum_{k=1}^{[M \sqrt{\ln n]}} \frac{(h(m)-1)^{k}}{k !} S_{k}(w) \\
& \quad \leqslant p(w)+\sum_{k=1}^{[M \sqrt{\ln w]}} \frac{(h(m)-1)^{k}}{k !} S_{k}(w)=v(m, w) .
\end{aligned}
$$

Из соотношений $(62),(63)$ и $(65)$ следует, что при $w \geqslant n \geqslant n_{3}$

$$
v(m, w)-v(m, n) \leqslant \max \left(\varepsilon_{3}(r, n), \delta_{3}(w)\right)(v(m, w)+v(m, n)) .
$$

В силу (64) и того, что $\delta_{3}(w) \rightarrow 0$ при $w \rightarrow \infty$, из последнего неравенства получаем, что при $n \rightarrow \infty, m \asymp n, w \geqslant n, w-n=o(n)$

$$
\limsup \left(\frac{v(m, w)-v(m, n)}{v(m, w)+v(m, n)}\right) \leqslant 0
$$

Из соотношений (58) и (66) следует (45). А из (45), как было отмечено ранее, следует (7). Лемма 1 доказана.

Положим в теореме $2 \beta=\sigma$ и при $t>0$

$$
\begin{aligned}
L_{1}(t) & =\exp (l(t)-\sigma \ln t), \\
L_{2}(t) & =\frac{\exp \left(x \sqrt{l(t)}+x^{2} / 2-\sigma \gamma\right)}{\Gamma(\sigma)} \\
r(t) & =\exp (l(t)) t^{\alpha} L_{2}(t)=t^{\alpha+\sigma} L_{1}(t) L_{2}(t)=t^{\alpha+\sigma} L_{0}(t),
\end{aligned}
$$

где $L_{0}(t)=L_{1}(t) L_{2}(t), \gamma$ - постоянная Эйлера, а функция $l(t)$ определена равенством (4).

В работе [33] доказаны следующие две леммы. 
Лемма 2. Пусть выполнено (3). Тогда для всякого фиксированного $\mu>0$ при $t \rightarrow \infty$

$$
f\left(e^{-\mu / t}\right)=l(t)-\sigma \ln \mu-\sigma \gamma+o(1)
$$

(функиия $f(s)$ определена равенством (17)).

Лемма 3. Предположим, ито имеет место (3). Тогда для некоторого $\alpha>1$ и произвольного $\lambda>0$ при $t \rightarrow \infty$

$$
\begin{aligned}
& \sum_{m=1}^{\infty} \exp \left(-\frac{\lambda m}{t}\right) m^{\alpha-1} \exp (l(t) h(m)) \\
& \sim \lambda^{-\alpha} \Gamma(\alpha) t^{\alpha} \exp \left(l(t)+x \sqrt{l(t)}+\frac{x^{2}}{2}\right) .
\end{aligned}
$$

Д ок а з а т ел ь с т в о т е о ре мы 1 . Согласно лемме 3 , выберем $\alpha>1$, для которого имеет место (68). Тогда для чисел $a(m, n)$, определенных в соотношении (10), и всех $u, v \in[0,1)$ в силу (16) и (17)

$$
A(u, v) \equiv \sum_{m, n \geqslant 0} m^{\alpha-1} a(m, n) u^{m} v^{n}=\sum_{m \geqslant 0} u^{m} m^{\alpha-1} \exp (h(m) f(v)) .
$$

Будем полагать в (69) $u=\exp (-\lambda / t)$ и $v=\exp (-\mu / t)$, где $\lambda, \mu, t>0$. Согласно (69) и лемме 2 , при $t \rightarrow \infty$

$$
A(u, v) \sim \mu^{-\sigma} e^{-\sigma \gamma} \sum_{m \geqslant 0} u^{m} m^{\alpha-1} \exp (h(m) l(t)) .
$$

Воспользовавшись леммой 3 , отсюда получаем, что при $t \rightarrow \infty$

$$
\begin{aligned}
A(u, v) & \sim \lambda^{-\alpha} \mu^{-\sigma} \Gamma(\alpha) e^{-\sigma \gamma} t^{\alpha} \exp \left(l(t)+x \sqrt{l(t)}+\frac{x^{2}}{2}\right) \\
& =\lambda^{-\alpha} \mu^{-\sigma} \Gamma(\alpha) \Gamma(\sigma) t^{\alpha} \exp (l(t)) L_{2}(t)=\lambda^{-\alpha} \mu^{-\sigma} \Gamma(\sigma) r(t)
\end{aligned}
$$

в силу (67). Из леммы 1 и последнего соотношения следует, что выполнены все предположения теоремы 2 . По этой теореме при $n \rightarrow \infty$

$$
\begin{aligned}
a(n, n) & \sim r(n) n^{-1-\alpha}=n^{\sigma-1} L_{1}(n) L_{2}(n) \\
& =n^{\sigma-1} L_{1}(n) \frac{\exp \left(x \sqrt{l(n)}+x^{2} / 2-\sigma \gamma\right)}{\Gamma(\sigma)} .
\end{aligned}
$$

Полагая в (10) $x=0$, отсюда получаем, что

$$
p(n) \sim n^{\sigma-1} L_{1}(n) \frac{\exp \{-\sigma \gamma\}}{\Gamma(\sigma)} \quad(n \rightarrow \infty) .
$$

Далее, из (10), (70) и (71) следует, что при $n \rightarrow \infty$

$$
\mathbf{E} \exp \left(\frac{\zeta_{n} x}{\sqrt{l(n)}}\right) \sim \exp \left(x \sqrt{l(n)}+\frac{x^{2}}{2}\right)
$$

или

$$
\mathbf{E} \exp \left(\frac{x\left(\zeta_{n}-l(n)\right)}{\sqrt{l(n)}}\right) \rightarrow \exp \left(\frac{x^{2}}{2}\right) \quad(n \rightarrow \infty) .
$$


Утверждение теоремы 1 следует из (2) и последнего соотношения. Теорема 1 доказана.

Следствие 1. Пусть выполнено (1). Тогда функиия $L(n)$ из (1) имеет при $n \rightarrow \infty$ следующий асимптотический вид:

$$
L(n) \sim L_{1}(n) \frac{\exp \{-\sigma \gamma\}}{\Gamma(\sigma)}=\exp \left(\sum_{k \in A, k \leqslant n} \frac{1}{k}-\sigma \ln n\right) \frac{\exp \{-\sigma \gamma\}}{\Gamma(\sigma)}
$$

\section{СПИСОК ЛИТЕРАТУРЫ}

1. Болотников Ю.В., Сачков В.Н., Тараканов В.Е. Асимптотическая нормальность некоторых величин, связанных с цикловой структурой случайных подстановок. - Матем. сб., 1976, т. 99, № 1, с. 121-133.

2. Болотников Ю. В., Сачков В. Н., Тараканов В. Е. О некоторых классах случайных величин на циклах подстановок. - Матем. сб., 1979, т. 108, № 1, с. 91-104.

3. Бендер Э.А. Асимптотические методы в теории перечислений. - Перечислительные задачи комбинаторного анализа. М.: Мир, 1979, с. 266-310.

4. Волынеи Л. М. Число решений уравнения в симметрической группе. - Вероятностные процессы и их приложения. М.: МИЭМ, 1985, с. 104-109.

5. Вольнеи Л. М. О числе решений уравнения $x^{s}=e$ в симметрической группе. Матем. заметки, 1986, т. 40, № 2, с. 155-160.

6. Волынеи Л. М. Пример нестандартной асимптотики числа подстановок с ограничениями на длины циклов. - Вероятностные процессы и их приложения. М.: МИЭМ, 1989, с. 85-90.

7. Grusho A.A. Properties of random permutations with constrains on the maximum cycle length. - Proceedings of the Third Petrozavodsk Conference on Probabilistic Methods in Discrete Mathematics (Petrozavodsk, 1992). Moscow/Utrecht: TVP/VSP, 1993, p. 60-63. (Progr. Pure Appl. Discrete Math., v. 1.)

8. Ивченко Г. И., Медведев Ю. И. О случайных подстановках. - Труды по дискретной математике. Т. 5, М.: Физматлит, 2002, с. 73-92.

9. Колчин А.В. Уравнения, содержащие неизвестную подстановку. - Дискретн. матем., 1994, т. 6, № 1, с. 100-115.

10. Колчин В. Ф. Случайные отображения. М.: Наука, 1984, 206 с.

11. Колчин В. Ф. О числе подстановок с ограничениями на длины циклов. - Дискретн. матем., 1989, т. 1, № 2, с. 97-109.

12. Kolchin V.F. The number of permutations with cycle lengths from a fixed set. Random Graphs (Poznań, 1989). New York: Wiley, 1992, p. 139-149.

13. Колчин В. Ф. Случайные графы. М.: Физматлит, 2000, 255 с.

14. Manstavičius $E$. On random permutations without cycles of some lengths. - Period. Math. Hungar., 2001, v. 42, № 1-2, p. 37-44.

15. Минеев М. П., Павлов А.И. О числе подстановок специального вида. - Матем. сб., 1976, т. 99, № 3, с. 468-476.

16. Минеев М. П., Павлов А.И. Об одном уравнении в подстановках. - Тр. МИАН, 1976 , т. 142 , с. $182-194$.

17. Павлов А.И. О числе и цикловой структуре подстановок некоторых классов. Матем. сб., 1984, т. 124, № 4, с. 536-556.

18. Павлов А.И. О некоторых классах подстановок с теоретико-числовыми ограничениями на длины циклов. - Матем. сб., 1986, т. 129, № 2, с. 252-263.

19. Павлов А.И. О подстановках с длинами циклов из заданного множества. - Теория вероятн. и ее примен., 1986, т. 31 , в. 3, с. 618-619. 
20. Павлов А. И. О числе подстановок с длинами циклов из заданного множества. Дискретн. матем., 1991, т. 3, № 3, с. 109-123.

21. Павлов А. И. О числе подстановок с конечным множеством длин циклов. - Тр. МИРАН, 1994, т. 207, с. 256-267.

22. Павлов А. И. Асимптотика числа подстановок с теоретико-числовыми ограничениями на длины циклов. - Докл. РАН, 1994, т. 335, № 5, с. 556-559.

23. Павлов А. И. О двух классах подстановок с теоретико-числовыми ограничениями на длины циклов. - Матем. заметки, 1997, т. 62, № 6, с. 881-891.

24. Постников А. Г. Введение в аналитическую теорию чисел. М.: Наука, 1971, $416 \mathrm{c}$.

25. Сачков B. Н. Отображения конечного множества с ограничениями на контуры и высоту. - Теория вероятн. и ее примен., 1972, т. 17, в. 4, с. 679-694.

26. Сачков $B$. Н. Случайные отображения ограниченной высоты. - Теория вероятн. и ее примен., 1973, т. 18 , в. 1, с. 122-132.

27. Сачков В.Н. Комбинаторные методы дискретной математики. М.: Наука, 1977, $319 \mathrm{c}$.

28. Сачков В.Н. Вероятностные методы в комбинаторном анализе. М.: Наука, 1978, $287 \mathrm{c}$.

29. Сенета Е. Правильно меняющиеся функции. М.: Наука, 1985, 141 с.

30. Shepp L. A., Lloyd S. P. Ordered cycle lengths in a random permutation. - Trans. Amer. Math. Soc., 1966, v. 121, №2, p. 340-357.

31. Тимашев $A . H$. Предельные теоремы в схемах размещений частиц по различным ячейкам с ограничениями на заполнения ячеек. - Теория вероятн. и ее примен., 2004, т. 49 , в. 4 , с. $712-725$.

32. Якьмив А. Л. О числе $A$-подстановок. -- Матем. сб., 1989, т. 180, № 2, с. 294-303.

33. Якьцив А.Л. О подстановках с длинами циклов из заданного множества. Дискретн. матем., 1989, т. 1, № 1, с. 125-134.

34. Якымив А.Л. О случайных подстановках с длинами циклов из заданного множества. - Вероятностные процессы и их приложения. М.: МИЭМ, 1991, с. 24-27.

35. Якымив А.Л. О некоторых классах подстановок с длинами циклов из заданного множества. - Дискретн. матем., 1992, т. 4, № 3, с. 128-134.

36. Yakymiv A.L. Limit theorems for random A-permutations. - Proceedings of the Third Petrozavodsk Conference on Probabilistic Methods in Discrete Mathematics (Petrozavodsk, 1992). Moscow/Utrecht: TVP/VSP, 1993, p. 459-469. (Progr. Pure Appl. Discrete Math., v. 1.)

37. Якымив А.Л. О подстановках с длинами циклов из случайного множества. Дискретн. матем., 2000, т. 12, № 4, с. 53-62.

38. Якымие $A$.Л. Распределение длины $m$-го максимального цикла случайной $A$ подстановки. - Дискретн. матем., 2005, т. 17, № 4, с. 40-58.

39. Якымив А.Л. Вероятностные приложения тауберовых теорем. М.: Физматлит, 2005,256 c.

Поступила в редакцию

24.XII.2005

Исправленный вариант

6.IX.2006 